\title{
Dark-Field Imaging based on Post-Processing of Electron Backscatter Diffraction Patterns in a Scanning Electron Microscope
}

\author{
Nicolas Brodusch, Hendrix Demers, and Raynald Gauvin \\ Department of Mining and Materials Engineering, McGill University, Montreal, Quebec, Canada.
}

Dark-field (DF) imaging can be performed by selecting a specific diffracted beam in the selected area diffraction pattern in conventional transmission electron microscope (CTEM) or in the convergent beam electron diffraction pattern in scanning transmission electron microscopy (STEM) mode [1]. The resultant micrograph provides high intensity of the objects in the probed volume that diffract in this particular direction. In contrast, dark-field micrographs can be obtained in STEM mode by capturing the signal from a specific range of scattering angles, with the most representative example being the high-angle annular dark-field imaging (HAADF) [2]. This leads to a contrast mostly based on atomic number differences between the different objects analysed [3].

These techniques were developed originally for CTEM and STEM. Because DF based on scattering angles is technically easy to obtain in a scanning electron microscope (SEM) by collecting the transmitted/diffracted signals with an electron detector below the thin specimen, it has been implemented in SEMs seriously since several years. This permitted taking advantage of the high contrast and low beam damage obtained at low accelerating voltages STEM in the SEM is now routinely achieved with a spatial resolution close to $1 \mathrm{~nm}$ in field-emission SEMs [4]. Despite these new possibilities, DF imaging only based on diffracted beams has not been achieved yet in a SEM.

The mostly used diffraction technique in the SEM has been, since the discovery of Venables [5], electron backscatter diffraction (EBSD) which has a spatial resolution of roughly 20-30 nm and which needs a limited bulk surface preparation compared to CTEM or STEM. EBSD is assumed to be related to the electron channeling pattern (ECP) diffraction technique by the reciprocity theorem [6], although its angular resolution is, at this time, limited by the pixel resolution of the acquisition equipment. Figure 1 is a comparison between an ECP and an EBSP acquired at $20 \mathrm{kV}$ from a [001] (001) silicon wafer. In this work, pseudo-Kikuchi patterns (EBSP) recorded via EBSD were stored and reprocessed by reporting pixels or clusters of pixels intensities from a specific location in a reference EBSP to reconstruct the final image (EBSD map). A resulting micrograph (called EBSD-DF image) was produced with a direct link to the diffracted beams in the EBSP and hence, to the crystallography of the sample, i.e., a DF image. The origin of the contrast is then similar to that of electron channeling contrast image (ECCI) as shown in Figure 2, in which EBSD-DF micrographs of an indented compressed iron specimen with different reflections are displayed. However, the post-acquisition processing is an invaluable advantage over ECCI because it allows generating multiple micrographs at the same time with only one set of EBSPs recorded in a beam raster fashion. This opens new ways of extracting and using the information contained in each EBSP and the main applications, at this point, are understanding deformation behaviors and interpretation [7] of channeling contrast [8]. 


\section{References:}

[1] D.B. Williams and C.B. Carter, Transmission electron microscopy: a textbook for materials science. 2009: Springer.

[2] S. Pennycook, Ultramicroscopy, 30 (1989), pp. 58-69.

[3] O.L. Krivanek et al, Nature, 464 (2010), pp. 571-574.

[4] P.G.Merli et al, Microscopy and Microanalysis, 9 (2003), pp. 142-143.

[5] J. Venables and C. Harland, Philosophical Magazine, 27 (1973), pp. 1193-1200.

[6] O.C. Wells, Scanning, 21 (1999), pp. 368-371.

[7] N. Brodusch, H. Demers, and R. Gauvin, Ultramicroscopy, 148 (2015), pp. 123-131.

[8] S. Kaboli et al, Journal of Applied Crystallography, (2015), Submitted.
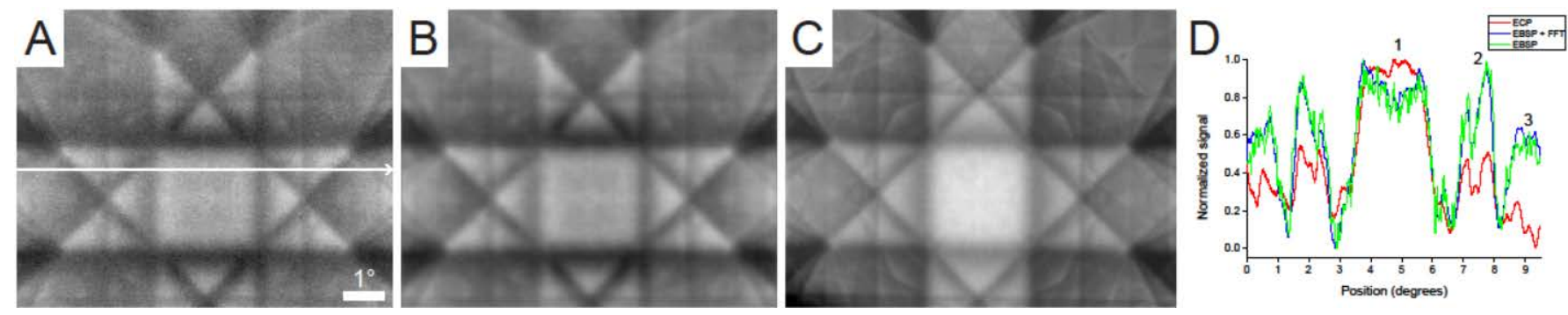

Figure 1. Comparison between an electron backscatter diffraction pattern (EBSP) and an electron channelling pattern (ECP) of a [001] (001) silicon wafer. (A) Raw and (B) digitally filtered EBSP and (C) ECP. Both were recorded with an accelerating voltage of $20 \mathrm{kV}$. The working distance was $10 \mathrm{~mm}$ for the ECP and the detector distance was $80 \mathrm{~mm}$ for the EBSP. Tilt angles were 0 and $80^{\circ}$ for the ECP and the EBSP, respectively. (D) Line profiles extracted from (A-C) show the higher resolution obtained with the ECP compared to the EBSP.
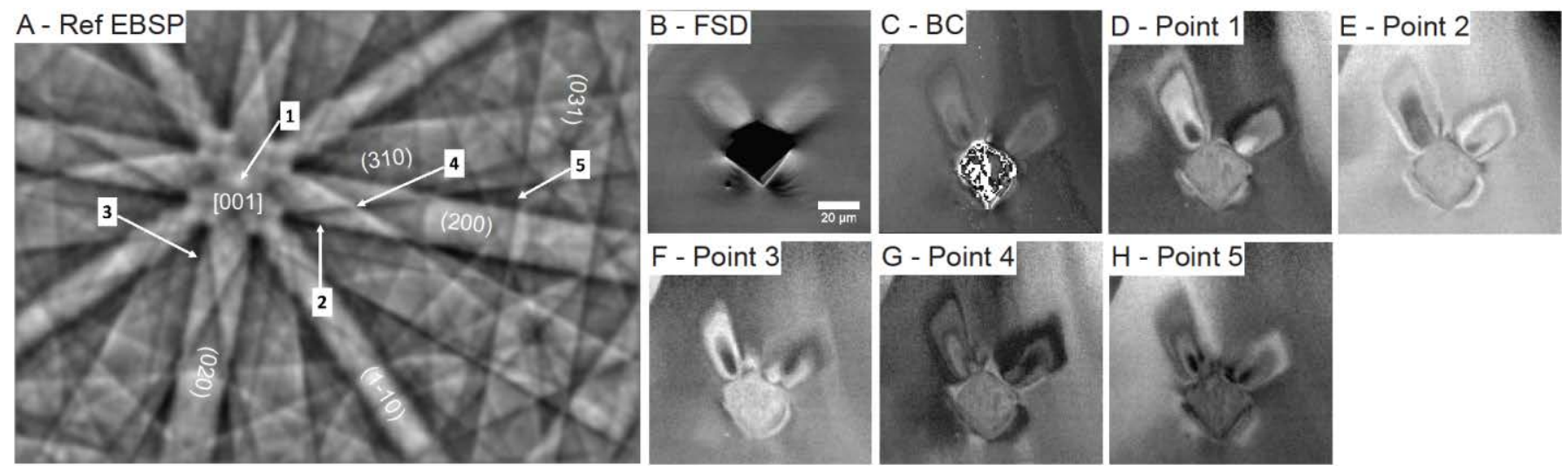

Figure 2. High contrast EBSD-DF images of a micro-hardness indent on compressed iron obtained using long EBSD detector distance for high angular resolution EBSPs with an accelerating voltage of $30 \mathrm{kV}$ and a detector distance of $50 \mathrm{~mm}$ as a function of the virtual beam position on the high angular resolution reference EBSP. (A) Reference EBSP, (B) FSD image, (C) band contrast (BC) map and (D-H) EBSD-DF images with specific reflections marked by arrows in (A). The EBSPs image resolution was $1344 \times 1024$ pixels. 